\title{
Research on TRIZ and CAIs Application Problems for Technology Innovation
}

\author{
Xiangdong $\mathrm{Li}^{1}$, Qinghai $\mathrm{Li}^{2}$, Zhonghang $\mathrm{Bai}^{2}$, and Lixiao Geng ${ }^{1}$ \\ ${ }^{1}$ Hebei University of Technology, School of Management, \\ Tianjin, P.R. China \\ ${ }^{2}$ Hebei University of Technology, Design Institute for Innovation, \\ Tianjin, P.R. China \\ \{Xiangdong.Li, Qinghai.Li, Zhonghang.Bai, Lixiao.Geng, \\ Lee96xd\}@163.com
}

\begin{abstract}
In order to realize application of invent problem solve theory (TRIZ) and computer aided innovation software (CAIs), need to solve some key problems, such as the mode choice of technology innovation, establishment of technology innovation organization network(TION), and achievement of innovative process based on TRIZ and CAIs, etc.. This paper shows that the demands for TRIZ and CAIs according to the characteristics and existing problem of the manufacturing enterprises. Have explained that the manufacturing enterprises need to set up an open TION of enterprise leading type, and achieve the longitudinal cooperation innovation with institution of higher learning. The process of technology innovation based on TRIZ and CAIs has been set up from researching and developing point of view. Application of TRIZ and CAIs in FY Company has been summarized. The application effect of TRIZ and CAIs has been explained using technology innovation of the close goggle valve product.
\end{abstract}

Keywords: Technology innovation, TRIZ, CAI, Manufacturing enterprises.

\section{Introduction}

With the quickening of global economic integration paces, the arrival of knowledge economy, cybereconomy, service economy and experience economy's era, the fast development of new industry such as information, competition at home and abroad has been becoming fiercer, and the manufacturing enterprises face the severe and complicated competition situation. Outstanding problem that the manufacturing enterprises meet is to lack key technologies, and lead to the fact of the market competition in inferior position. Meanwhile, the manufacturing enterprises have numerous profound contradictions and problems to need to be solved. One of the core and key problems among them is technology innovation question.

The technology innovation means enterprises use innovative knowledge, new technology, new process, and adopt new mode of production and management to improve product quality, develop and produce new products, offer new service, occupy the market and realize market value [1]. At the earliest, Austrian-born American economist Joseph A. Schumpeter puts forward the concept of "innovation", and opens the 
innovative theory. He has offered analytical framework for explaining technology innovation and economic development. After that, numerous scholars carry on research from different angle to technology innovation, including a great deal of respects, such as technology innovation strategy, process, mechanism, key elements, mode, organization, function, ability, policy, etc.. But, it still need to further make an intensive study of applying TRIZ that how to enhance technology innovation ability of the Manufacturing enterprises.

Enterprise's technology innovation ability includes the decision ability of technology innovation, research and development ability, engineered ability, production capacity, market exploring, organizing and coordinating ability and resource distribution ability, etc.[2]. In order to improve the technology innovation ability of the Manufacturing enterprises, make TRIZ bringing into fullplay in the Manufacturing enterprises, the pivotal things are to choose suitable technology innovation mode, set up perfect TION and form the high-efficient innovative process.

At present, the research, popularization and applying of TRIZ are more and more arousing the attention from countries all over the world. In Russia, TRIZ theory is regarded as compulsory subject of professional technique of university, and has already applied to project field extensively. Some universities of U.S.A. have offered TRIZ courses, and have established the research and advisory organization about TRIZ. Since 1990s, some famous companies such as General Motors, Chrysler, Xerox, Rocco and Will and Johnson, etc. have already begun to study and apply TRIZ method, and have reported about the case of success. In Europe, the creative research and development activities centered on Royal Institute of Technology of Sweden (KTH) are carried on through concentrating a dozen enterprises on beginning to utilize TRIZ. Many magazines are introducing TRIZ theory and application case constantly since 1996 in Japan, and the books of TRIZ begin to be published too[3].

TRIZ and CAIs based on TRIZ[4,5,6] can give knowledge of different field. During the process of innovation of enterprises, CAIs is an efficient tool.

The Manufacturing enterprises distribute in each field extensively to meet diversification, individualized consumption demand. As an important and active strength in national economic development, development and growth of the Manufacturing enterprises are of vital importance. To study the Manufacturing enterprises' technology innovation behavior and improve technology innovation ability are also of vital importance. Therefore, key problems and practice application of applying TRIZ technology to the Manufacturing enterprises and raising technology innovation ability of it is researched in this paper.

\section{Demands to TRIZ and CAIs of the Manufacturing Enterprises}

In the era of knowledge-driven economy, technological progress and technology innovation have already become the most outstanding factor of restricting development of the manufacturing enterprises[7]. In order to meet the new fiercer market environment at home and abroad and competition rule, the key enhancing core competitiveness of the manufacturing enterprises is to improve their own technology innovation ability. J. A. Schumpeter think only leading invention into the production system, it could become innovation. When people invent and create, solve the technological 
difficult problem, and realize the technology innovation, can follow certain scientific method and rule, can realize new invention and creation or solve technological difficult problem rapidly, and realize the technology innovation. One of this new methods and rules is the TRIZ and the application of CAIs. The development of modern economy indicates the Manufacturing enterprises have stronger adaptability to the technology innovation. As invent problem solve theory, among enterprise technology innovation activity, choose and utilize suitable method and tool of TRIZ, can offer the scientific and effective technical innovation means for the Manufacturing enterprises, and have important impetus and promoter action to the Manufacturing enterprises' innovation ability improvement and innovative result's obtainment especially.

Through the development for many years, TRIZ has already become a theory and method system of solving the innovative practical problem, and is test by practice. Meanwhile, a lot of companies regard TRIZ as the key principle to develop computer aided innovation (CAI) software, in order to support the innovative process, such as TechOptimizer software of the Invention Machine Company of U.S.A. and TRIZSoft software of the Ideation International Company, etc.. CAI software become effective tool solving technological difficult problem and realizing innovation of overseas enterprises and the most advanced technical field already. TRIZ theory already got the attention of the academia and business circles in China. For instance, Design Institute for Innovation of Hebei University of Technology always dedicated to the research of TRIZ theory and practice application. Have developed first set of CAI software"InventionTool" based on TRIZ with independent intellectual property right, and realized commercialization. It has been applied to Shijiazhuang No.1 Valve Factory Co. Ltd., Baoshan Iron and Steel Co. Ltd., Emerson Company, etc.. Therefore, TRIZ is not merely the innovative tool of the big enterprises, can become the tool of technology innovations of the numerous Manufacturing enterprises even more. Through training talented person mastering TRIZ, obviously improve the innovative levels of the Manufacturing enterprises. By establishing and perfecting the technology innovation system, form the good innovative environment for the Manufacturing enterprises gradually.

\section{Key Problems of TRIZ and CAIs Application during Technology Innovation Process}

The technology innovation refers to the technological economic activity related to research and development of new technology (including new products, new craft), production and commercialization [8]. It is the entrepreneur that catches the potential profit chance of market, regards obtaining commercial interest as the goal, and sets up the system of production and management with stronger, higher efficiency and lower expenses. And to put out new products, new production technology method, open up new market, get new raw materials or semi-manufactured goods or sets up new enterprise organization. It is the total process including a series of activities such as the science and technology, organization, commerce and finance, etc.[9]. That is why the factors influencing enterprise's technology innovation include the technological factors, and also include the non-technological factors. The non-technological factors includes respects such as the system, management, fund, corporate culture and spirit 
of entrepreneurship, etc.. As to the technology innovation of the Manufacturing enterprises, on one hand fully give play to the role of non-technological factors, on the other hand should utilize the technological factors to produce the positive innovative behavior. During the technology innovation process of the Manufacturing enterprises, from applying TRIZ and CAIs point of view, the key problems of technology innovation is the choice of the innovative mode, establishment of technology innovation organize network and realization of the innovative process based on TRIZ and CAIs.

\subsection{Mode Selection of Technology Innovation}

The technology innovation mode refers to technology innovation way, method. According to the technology innovation method, different source which enterprises obtain technological resources and technological ability, the technology innovation is divided into three kinds of modes: independent innovation mode, cooperative innovation mode and imitating innovation mode.

From enterprises point of view, independent innovation is a kind of technology innovation activity that enterprise independently carries on regarding researching and developing as foundation. To realize the commoditization of technology finally, obtain the commercial profit. The types of independent innovative mode mainly include primitive innovation, integrated innovation, re-innovation after introducing, digesting and assimilating. Cooperative innovation refers to a kind of resource-sharing and complementary advantages technology innovation mode among enterprise, institution of higher learning and scientific research organization or among enterprises. It is a kind of union technology innovation behavior through the common input, participation of the cooperative parties, to realize achievement-sharing and shared risks. Imitating innovation is a kind of advancing technology innovation behavior. Through grasping and absorbing the key technology which take the lead in the innovator, invest research and development strength to carry on further development and produce the competitive products. Compare the above three kinds of different technology innovation modes, the technology innovations of enterprises are set up on the foundation of inside and external factors acting on commonly. Making use of external factors to realize cooperative innovation and imitating innovation; making use of internal factors to realize the independent innovation of new products and new crafts.

Looking from developed course of the technology innovations of various countries, the technology innovation mode has undergone such an evolvement orbit on the whole: from imitating innovation to cooperative innovation, to independent innovation. The final purpose of technology innovation evolvement is to impel enterprises to form independent innovation ability continuously, and train enterprise's lasting market competition advantage [10]. The advantage of independent innovation is obvious: first of all, help to be in leading position technically, form monopolizing or relative monopoly position through the technological barrier; secondly, the great innovation of key technology can drive the production of a series of new products; finally, in leading position on the market, enterprise can obtain monopoly profits [8]. The demand of independent innovation mode are very high in some respects, such as research and development ability, achievement accumulation, technological position, invest, risk ability, etc. 
For most Manufacturing enterprises, the material foundation condition of technology innovation and technological ability are relatively weak because the technician is scarce, fund and information resources shortage. If totally launch the innovative behavior with one's own strength, it is very difficult to improve the technology innovation level and innovation ability shortly. It is a kind of wise action to advance enterprise's technology innovation together by innovation of cooperation with other departments.

Though TRIZ and CAIs has put emphasis on regularity of invention and stylized process, but often study at the beginning, there are greater difficulties on training and practical application. It requires users to possess abundant field knowledge [11]. Because of the technicians of the Manufacturing enterprises at present, the shortage of the technician who especially masters TRIZ and CAIs, the Manufacturing enterprises need a quite long studying and training course effectively to grasp and use TRIZ and CAIs. Proceed from the angle of applying TRIZ and CAIs in the manufacturing enterprises, enterprise need to carry on close longitudinal cooperation with institute of higher learning and TRIZ research institution, introduce TRIZ and CAIs in the technology innovation process of enterprise, and make the manufacturing enterprises realizing optimizing resource distribution, improve innovative efficiency and result, thus promote the further improvement of enterprise's innovation ability.

\subsection{Establishment of the TION of Applying TRIZ and CAIs}

At present, the technology innovations of enterprises generally go on inside and outside two aspects. First of all, research and development organization should be set up inside conditional enterprises independently, and make enterprises become the real subject of technology innovation. Secondly, because the independent research and development strength of enterprises is not strong, need to strengthen the cooperation with the outside research and development organization, and implement production, teaching, research combination together. At the present stage, proceed from the angle of saving the fund and rational utilizing existing talent resource, prompting enterprises and $R \& D$ organization to integrate are required by circumstances[1]. The technology innovation can be realized through organization network. Therefore, in order to meet the needs of cooperative innovation mode of the Manufacturing enterprises, should set up an open TION of enterprise leading type, strengthen organizations the cooperation among the enterprise, institution of higher learning, scientific research institutions, etc., and also between enterprises and enterprises.

Under the circumstances of market and technological competition fiercer day by day, TRIZ and CAIs is popularized and applied rapidly in recent years. TRIZ and CAIs theory and method apply to technical field extensively already. TRIZ and CAIs gives play to important function in solving enterprise's technology innovation, especially in the products innovation question. It is popularized and brought enormous income in a lot of trans-corporations rapidly. Because strong function of TRIZ theory and CAIs in the innovative process, research and practice of TRIZ and CAIs can be developed rapidly in worldwide. The research center of TRIZ has been established in countries such as Russia, Sweden, Japan, Israel, U.S.A.,etc.. In China, TRIZ research center of Hebei University of Technology has also been established. Through using the TION, centering on the research and development organization of enterprise, 
absorbing TRIZ research centers of the institution of higher learning, scientific research institution, etc. to enter the innovative network, giving play to intelligence of TRIZ expert, and cooperating innovation, to improve technology innovation ability of the Manufacturing enterprises.

\subsection{The Innovative Process on the Basis of TRIZ and CAIs}

A core and basic link of technology innovation is the research and development (R\&D), namely products innovation. For surviving and developing, according to the flexible characteristic of product change, the technology innovation of the Manufacturing enterprises should be put in products innovation especially, in order to make and seize the first chance of the market. Through TRIZ improving the studied and developed result, realizing the process of products innovation based on TRIZ, it is an important means and ways to improve enterprise's technology innovation ability.

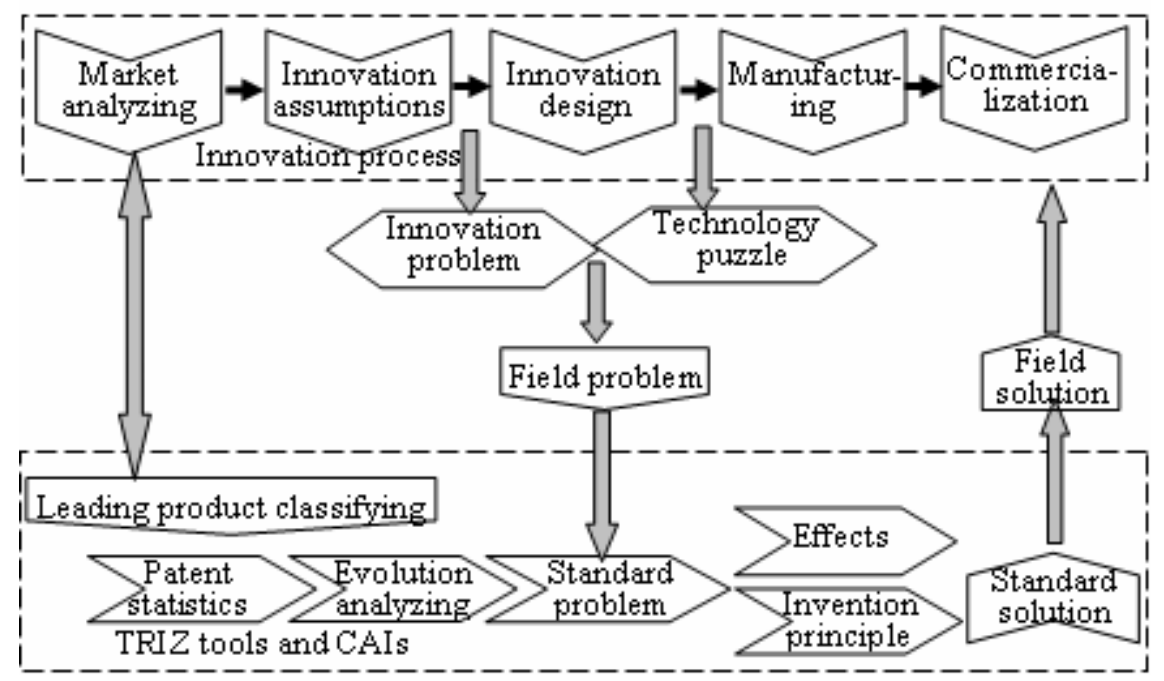

Fig. 1. Innovation process based on the TRIZ and CAIs

During the technology innovations of enterprises, the key lies in that there is combination of innovative process now existed in enterprises with TRIZ and CAIs. Fig. 1 describes the process of TRIZ and CAIs to support enterprise's products innovation. Through market analysis, to carry on the leading product classification of enterprises product family; and then carry on patents count, go on technology evolution analysis in terms of quantity, rank, etc., predict the future development trend of innovation, confirms evolving and innovative direction. According to innovative problem and technological puzzle appearing in the innovation imagine stage of the fuzzy front, design and produce stage, to form the field problem. Changing the field problem need to be solved into TRIZ standard problem, next using TRIZ tool of CAIs, such as the invention principle and effect, etc. to obtain the standard solution of the problem. Finally, changes it into the domain solution, to form the innovation result. 


\section{TRIZ and CAIs Application Practice of the FY Company}

As the tool of promoting enterprise's technology innovation ability raised, TRIZ and CAIs has offered the strong theoretical direction for innovation, research and development of enterprises. Apply it to innovative practice of FY enterprise, can make the key competitiveness of enterprises obviously improved. In view of the technology innovation demand of the FY company, according to its production and management and products characteristic, adopt the support of cooperative innovation mode and innovation network, it is that enterprises have brought the enormous income to apply the innovative process based on TRIZ and CAIs.

\subsection{Mode Adopting of Cooperative Innovation}

FY company is a nonstandard valve specialized factory. The TRIZ research center of Hebei University of Technology has been studying to TRIZ and CAIs for many years, and has formed characteristic of "the integrating type technology innovation and software development based on TRIZ". Have established close cooperation with many enterprises, and offer the theory and technical support for technology innovation management and research and development of new products of these enterprises. Proceeds from technology innovation strategy angle of it, FY company carries on the cooperative innovation of production, teaching, research with the TRIZ research center of Hebei University of Technology, have obviously improved the technology innovation strength of the company.

\subsection{Establishment of the TION of Enterprise Leading Type}

The technology innovation and cooperation of FY company goes on two aspects of enterprise's inside and outside. First of all, enterprise sets up research and develop organization independently--Technology department, which becomes technology innovation main part of enterprise. Meanwhile, under the support of the government scientific and technological departments, has set up the valve engineering research center at city level inside. Secondly, because of under strength of autonomous innovation of enterprise, from saving fund, and rationally utilizing enterprise's existing talent resource angle, cooperates with organizations, such as the TRIZ research center of Hebei University of Technology, etc. longitudinally, has jointly established the research base of "production, teaching, research", and has sets up valve research institute in Hebei University of Technology. Through introducing TRIZ and CAIs expert and technology, FY company improves enterprise's technology innovation ability.

By the support of fund and policy of the government, and close cooperation between FY company with institution of higher learning, realized longitudinal cooperative innovation. Unite other designing institutes at the same time, research institutions such as enterprises, etc., established of the TION of enterprise's leading type. This network usually still includes different subjects of a lot of levels and types, such as government, intermediary and financial institution, etc. FY company is core enterprise, which locates in the main part position. On one hand core enterprise has direct or indirect contacts with other networks member and interact, on the other hand, every member is carrying on the exchange of material and energy with the external environment constantly, form 
open and dynamic operation way. Through absorbing the TRIZ research center to enter the network, has promoted efficiency and result of enterprise's technology innovation, and have promoted the benign development of the network.

During the cooperative innovation, applying the TRIZ and CAIs, solve the significant, pivotal and urgent technological problem and research and development of new products in valve industry especially. The purpose lies in promoting the fast development of enterprise and improvement of valve industry overall engineering level.

\subsection{Application of Innovative Process Based on the TRIZ and CAIs}

Combining the TRIZ and CAIs and enterprise's existing innovative process, can great improve the technology innovation results of enterprise. Under the support of the TION of FY company, considering the application demand that is expanding constantly, in order to develop the high-quality valve products which meet market demand as soon as possible, the innovative project team has been set up, which is made up of the technology department, sales department of the FY company and the TRIZ research center of Hebei University of Technology. The technology department locates in the main part position among them. Through finding the potential problem in time that enterprises exist in the technology innovation currently, according to the support of enterprise's products innovation process from the TRIZ that Fig. 1 provides, apply TRIZ to the innovative process of cooperation of the company.

First of all, to carry on classification of general product family according to the leading valve products of enterprise, including butterfly valve, brake valve, electric blind plate valve, check valve, close goggle valve, etc. General product family of enterprise can include products view of different phases, project manual, products standard, engineering change sheet and review sheet, etc. which form general product family view. Meanwhile, according to market survey and demand analysis of the sales department, the key innovative products are defined. For example, the technology innovation activity of the product series of the close goggle valve have been launching in 2007.

Through searching for patents of the close goggle valve, and applying TRIZ's technology evolution theory, technology evolution trend analysis has been launched in terms of quantity, rank, etc. The future development trend of the close goggle valve was predicted, and its evolving and innovative direction was confirmed. Such as one of evolving and innovative direction is technology innovation of asymmetry clamping sealing device. Then, the innovative puzzle problem of this field of asymmetry clamping sealing device was expressed as TRIZ standard problem. Under the support of CAIs "InventionTool", the standard solution of the problem was got through applying dynamical invention principle and asymmetry invention principle of TRIZ's invention principle tools. Finally, the technology innovation achievement was formed by changing field solution. The adoption of this technology makes the sealed ability of the close goggle valve hike up, ability of bearing pressure raise 2.4, and make valve can bear external pipeline force (At present, valve can be unable to bear the external force of the pipeline in home and aboard.). In addition, by using amalgamating invention principle, the volume of valve inner parts was decreased markedly. Adopting the dimension change invention principle, mechanical performance and service life of the valve has been improved. At present, the cooperative innovation achievement of this 
valve has already applied for the invention patent. This patented product will be put on market in 2008, Thus, realize the whole process of technology innovation by using TRIZ and CAIs.

\section{Conclusions}

According to the characteristics and existing problem of the manufacturing enterprises, the demands for TRIZ and CAIs of the manufacturing enterprises have been analyzed in detail. The factors influencing enterprise's technology innovation are explained, it includes two respects: technical and untechnical factors. Proceed from the angle of applying TRIZ and CAIs in the manufacturing enterprises, points out the technology innovation mode choice, the establishment of TION and the realizing of innovative process based on TRIZ and CAIs to be the key of the success of the manufacturing enterprises' technology innovation. Three kinds of technology innovation modes of independent innovation, cooperative innovation and imitating innovation have been analyzed. In order to improve innovative efficiency and result and overcome scarce shortcomings of talents who master TRIZ and CAIs technology of the manufacturing enterprises, the viewpoint of fully utilizing cooperative innovation to make the manufacturing enterprises generate the positive innovative behavior is clarified. Need to set up an open enterprise leading type TION, and strengthen the longitudinal cooperation of organizations such as the manufacturing enterprises and institution of higher learning, etc. According to the process solve problem of TRIZ and CAIs, combining the general innovative process of enterprises from researching and developing the angle, the process of technology innovation based on TRIZ and CAIs have been set up. From the adopting of cooperative innovation mode, the establishment of enterprise's TION of leading type and the using of innovative process based on TRIZ and CAIs, explained practice application of TRIZ and CAIs in FY company and the application results of TRIZ and CAIs with technology innovation of the close goggle valve product. Based on the analysis of this paper, TRIZ and CAIs technology has offered an effective and powerful means for the technology innovation of the manufacturing enterprises. The goal of sustainable development of enterprises can be realized by using TRIZ and CAIs.

\section{Acknowledgements}

This research is supported in part by the Key Project of the Ministry of Science and Technology of the People's Republic of China under Grant Numbers 2008IM030100, and the science and technology key project of Hebei Province under Grant Numbers $09212102 \mathrm{D}$.

\section{References}

1. Zhao, J.D., Wang, S., Wang, S.Y.: Strategy choice of technology innovation of current enterprises at home (in Chinese). Productivity Research, 125-126 (2007)

2. Yu, G.B.: Countermeasure of enhancing technology innovation ability of enterprises at home (in Chinese). Advanced Materials Industry, 75-77 (2007) 
3. Information center of science and technology in Heilongjiang province. TRIZ introduction reading under guidance (2007-10-22/ 2008-01-07),

http://210.76.36.129/3.asp?ArticleID $=493$

4. Altshuller, G.S.: The Innovation Algorithm. TRIZ, Systematic Innovation and Technical Creativity, Worcester: Technical Innovation Center, INC (1999)

5. Tan, R.H.: Theory of Inventive Problem Solving (in Chinese). Science Press, Beijing (2004)

6. Savransky, S.D.: Engineering of Creativity. CRC Press, New York (2000)

7. Qiu, N.Z.: Problem research of technology innovation of the small and medium-sized enterprises (in Chinese). Coastal enterprises and science \& technology, 22-24 (2007)

8. Li, J.Q.: Mode adopting of technology innovation of the small and medium-sized enterprises (in Chinese). China High Technology Enterprises 4, 49-51 (2006)

9. Fu, J.J.: Innovation science of technology. Tsinghua University Press, Beijing (1998)

10. Cao, F., Yang, N.N.: Path choice of technology innovation of the small and medium-sized enterprises in industry evolution-take the information industry (in Chinese). Industrial Technology \& Economy 26, 18-22 (2007)

11. Wang, J.Q., Wen, Z.Z., Sun, H.L.: Comparative study of creative design method-TRIZ theory and BS method (in Chinese). Machinery 41, 7-9 (2003) 